\title{
Critical Determinants of Cytokine Storm and Type I Interferon Response in COVID-19 pathogenesis
}

Selvakumar Subbian ${ }^{1}$ and Santhamani Ramasamy ${ }^{1}$

${ }^{1}$ Public Health Research Institute (PHRI) at New Jersey Medical School, Rutgers University

October 26, 2020

\section{Hosted file}

MEDRXIV-2020-216432v1-Subbian.pdf available at https://authorea.com/users/364742/articles/ 488941-critical-determinants-of-cytokine-storm-and-type-i-interferon-response-in-covid19-pathogenesis 